\title{
Tenuta: Strategies for Providing Guidance on Usability and Accessibility
}

\author{
Nigel Bevan ${ }^{1}$, Helen Petrie ${ }^{1}$, and Nigel Claridge ${ }^{2}$ \\ ${ }^{1}$ Department of Computer Science, University of York, Heslington, York YO10 5DD \\ ${ }^{2}$ Scandinavian Usability Associates, Angantyrvägen 11, Djursholm, Sweden 18254 \\ tenuta@cs.york.ac.uk
}

\begin{abstract}
The EU Tenuta project was funded to assist EU eTEN projects improve the usability and accessibility of new e-services that are being validated on a European scale. The challenges facing Tenuta included how to initially convince projects that these were important issues, how to gain the confidence of projects that they would benefit Tenuta assistance, and how Tenuta could make an effective contribution with only a few days support available to each project. The eventual strategy was to arrange activities such as training courses where we made personal contacts with projects, which resulted in being invited to provide on-site support. We carried out usability and accessibility evaluations before visiting projects, and reported the results as part of a two to three day workshop with the project, which included assistance with redesign, and a roadmap for future activities. Projects gave very positive feedback on the benefits obtained.
\end{abstract}

\section{Introduction}

The European Union (EU) eTEN programme [3] supports the roll-out of e-services across Europe, based on the results of previous EU-funded research projects. EU research projects tend to be technically driven, so usually give little consideration to the usability and accessibility of the e-services. But the eTEN programme is intended to validate use of the e-services in different markets, so is much closer to providing a commercial service, where usability and accessibility should be key issues.

The objective of the EU Tenuta support action [5] is to improve the usability and accessibility of these EU e-services, by providing guidance on how to design systems that are both accessible and usable.

Currently accessibility is primarily concerned with sensory, physical and cognitive compatibilities, while usability is primarily concerned with cognitive compatibility. In most organizations they are the responsibility of people with different skills (technical and human). Neither approach alone is sufficient. Only by integrating these approaches in e-service development can disabled and older be given increased autonomy to play a full role in society.

Many organizations pay lip service to ease-of-use without employing any specific methods to ensure that it is achieved. Many web developers know that web 
accessibility is important, but many do not understand the intricacies of the Web Content Accessibility Guidelines [8] developed by the Web Accessibility Initiative (WAI) of the World Wide Web Consortium and how to apply them.

\section{Tenuta Support Action}

A paper at HCI International 2005 [1] reported the objectives of the Tenuta project and the challenges that it faced. We anticipated that the main problem would be to provide effective support within the constraints of very limited resources of between 3 and 10 person days of on-site assistance for each project supported.

The eTEN programme management expected that offering free usability and accessibility support would be popular with eTEN projects, but the first announcement of our services by eTEN met with no response. What we gradually learned was that although most projects had little experience of usability and accessibility, they were reluctant to invite an unknown third party to assist them, even one endorsed by the eTEN programme management. Almost all projects that we have now assisted had prior personal contact (e.g. via one of our training courses) or personal recommendation.

So our strategy was to find opportunities to meet project members, by attending meetings and workshops, and organizing training courses. Projects were much more willing to initially commit to sending people to a two or three day training course, than committing to even one or two days on site support. Following attendance at the course, many projects requested more specific support.

\subsection{Training Courses}

The objective of the two-day training course was to familiarize participants with usability and accessibility issues, and give them some training in basic techniques that they could use themselves.

The accessibility day started by explaining the characteristics and needs of disabled e-service users, followed by a demonstration of use of a screen reader by a blind user navigating a web site. Many participants commented that this gave them a real insight into the problems encountered by blind users.

The course explained the $\mathrm{W} 3 \mathrm{C}$ guidelines [8], and gave participants the opportunity to try using tools [7] to check existing web sites for conformance with the guidelines. However, we emphasized that accessibility could only be achieved by working with users to understand how they interact with e-services and by undertaking some user testing.

For the usability day, it was assumed that the audience should already have a good understanding of user requirements for their project, so the emphasis was on how to use this knowledge to produce appropriate designs. The course included an explanation of the business case for user centered design and an overview of how user centered design methods can be used during development.

We had to decide what to focus on for the main content. One approach is to teach heuristic evaluation principles and give participants experience of carrying out heuristic evaluations. This has the advantage of helping developers understand how 
basic usability principles can be incorporated into design. But it was felt to be too ambitious for a short course.

We decided instead to teach people how to carry out simple user testing. A stepby-step process was defined for planning and carrying out a user-based evaluation. Emphasis was placed on the need for users to carry out realistic tasks, and for the observer to only provide assistance as a last resort. Simple user testing could potentially enable developers to identify major usability issues with the minimum of training. One objective of this part of the course was to convey the message that although users might be quite satisfied after having seen a product demonstrated, they may find that they are totally unable to use the product on their own.

Most of the participants' implementations were web-based, so we also introduced them to the guidelines for effective web design and usability for information-oriented sites produced by the U.S. Department of Health and Human Services [6]. These guidelines have the advantage that they have been through a thorough review process, are simply described with examples, and can either be purchased as an inexpensive book, or downloaded free as a pdf. Having explained some typical guidelines, we showed how these could be used to evaluate existing web sites. These guidelines should provide a valuable learning resource for participants after the course.

The course has received very positive feedback, although it appears that some participants still thought there should be simple rules they could follow to make a site usable.

\subsection{On Site Support}

The overall approach to providing support was:

1. An initial telephone conference to review the needs for usability and accessibility, to clarify the intended context of use of the e-service, and to decide how Tenuta could provide the most effective form of support.

2. A review of the usability and accessibility of the e-service, carried out remotely, in advance of a visit.

3. In most cases, a 2-day on-site workshop to give a brief introduction to usability and accessibility, followed by presenting the results of the accessibility and usability reviews and recommendations for design improvement and discussing a road map for continued review.

4. Follow-up activities to support implementation of the recommendations.

\subsection{Context of Use}

An essential prerequisite for usability and accessibility work is to have a clear understanding of the:

- Intended user groups for the project e-service.

- Reasons that each user group is expected to use the project e-service.

- Context in which this will happen.

- Tasks that each user group will perform.

- Relative importance of the user groups and tasks.

- Importance of supporting users with disabilities. 
Although eTEN projects should already have a clear understanding of their potential users, our task is to help projects stand back and evaluate their solutions from the perspective of their users.

\subsection{Expert Evaluation}

Having gained basic information on the intended context of use from an initial telephone discussion, Tenuta consultants carried out an expert-based evaluation of the usability and accessibility of the project solutions. This was based on established usability principles, web design guidelines (such as [6]), established accessibility principles (the Web Content Accessibility Guidelines [8]) and testing the solution with a range of assistive technologies (particularly screen readers used by blind people, screen magnification programs used by partially sighted people and support software used by dyslexic people). It included both detailed aspects (such as screen design, terminology and support for navigation) and broader task and user support issues: does the project solution support specific user groups in achieving their goals? The effort required (including producing a report) was 1.5-2 person days by an experienced consultant for usability evaluation, and about 3 days by an experienced accessibility consultant to check a sample of 10 pages against the 10 most commonly violated WAI checkpoints [2, 4, 8] and conduct some testing with assistive technologies.

\subsection{On-Site Workshop}

To maximize the participation by project members, where possible an on-site workshop was arranged. The prime objective of the workshop was to help the project understand how to improve the usability and accessibility of their e-service. It started with a brief introduction to usability and accessibility.

Next, we stepped though the project e-service, discussing the usability and accessibility problems that have been found, and their potential solutions. At this point it is important to gain the support of the project members, so that we can help them implement their own solutions, rather than appear to be imposing a solution on them.

The meetings were most successful when we were working with a group of developers who were in a position to implement the conclusions in a planned new version. We have had most success when our meeting with the project has been hosted by the developers with whom we can work to implement the results of our findings. With one project we only had half a day to present to a project management group who seemed reluctant to accept the implications of the usability findings. The review of usability often raises wider issues of the extent to which the real user needs are understood: why will people use the project e-service, and can use be sustained after the end of eTEN financing?

Feedback that we received early on and incorporated into later workshops included providing a structured methodology for usability testing, and references to further information that could be followed up by projects.

If many cases we went on to assist the project in creating plausible design solutions to the problems that have been identified and agreed. 


\subsection{Follow-Up Activities}

One problem with providing assistance on a consultancy basis is that it is not always clear whether the project has acted on our findings. Sometimes the project management is driven by meeting pre-planned deadlines, and is unwilling to accept the risks they run by proceeding with an unusable solution.

\section{Example Case Studies}

\section{1 eParticipate}

eParticipate is creating a European service based on experience in the UK of assisting Local Authorities in the effective use of multimedia and webcasting to create and deliver audiovisual content that really makes an impact in local communities.

To assess the accessibility of the webcasting eService, it was decided that testing by users with disabilities who use assistive technology was vital, because of the use of an embedded media player in the service. Such a service can (and has) been made accessible in other projects we have advised, but to assess the real accessibility of the solution goes far beyond the specificity of the current WCAG accessibility guidelines. The particularly difficult case is whether the solution will be accessible to screenreader users.

Therefore two highly experienced screenreader users were recruited to test the solution, who could make most use of the functionality of their screenreader, and possibly try different methods for accessing the webcasts. If these users could successfully use the solution then it could subsequently be tested by less experienced screenreader users and users of other assistive technologies.

A testing protocol was established by Tenuta in collaboration with the eParticipate team, to ensure that all the key functionalities of the solution would be assessed for accessibility. This took the form of a scenario of five tasks that the users would work through, trying different tasks with the webcasting system.

Two highly experienced users, one who uses JAWS and one who uses WindowEyes were recruited to take part in this initial testing. The JAWS user worked through the scenario, but could not access the webcast at all. Neither could he access some of the information in the titles to the webcast which the team had thought might be accessible. The WindowEyes user had to cancel his session due to family problems, but given the problems by the JAWS user, it was decided to postpone the session with the WindowEyes user until more work has been done on the accessibility of the solution based on the results so far. The JAWS user has offered to re-test the solution remotely when further work has been undertaken.

\subsection{EuroWorkSafe}

EuroWorkSafe provides information, services and a database of resources related to occupational cancer risks and prevention. The project has carried out a thorough analysis of user requirements, and identified clear scenarios of use for medical, professional and educational users at different sites around Europe. 
Tenuta representatives visited the project for two and a half days soon after the first trial version of the web service was available.

Tenuta provided brief training in usability and accessibility, and then reported on the results of a usability and accessibility review of the site. Several changes would be required to the current site to make it accessible to users with disabilities, so it was agreed to help the project ensure that the next version of the site was accessible and then carry out a full accessibility audit.

The main issues revealed by the usability review were that there were some inconsistencies in navigation, that some features of the site are difficult to find and that some technical terminology might not be familiar to the intended users.

Detailed discussion of the users' objectives in coming to the site suggested three main purposes: looking for general information on occupational cancer, searching for specific resources related to occupational cancer, or interest in consultancy services provided by EuroWorkSafe. The EuroWorkSafe project proposed a new navigation structure to more directly support these tasks, which also resulted in a more consistent approach to navigation. This will be implemented in the next release.

Tenuta also recommended that EuroWorkSafe observes three to five representative users using the new site, individually carrying out typical tasks without any assistance. Similar user testing of the current site could also help inform the new design.

\subsection{Medical Web Site}

Tenuta reviewed the usability of a web service that provides information for doctors that is not easily available elsewhere. The project had demonstrated their web solution to doctors and incorporated some feedback. The project was visited by Tenuta shortly before a planned validation in several countries by 500 users.

The usability review suggested that it is unlikely that any experienced web user who makes the effort to familiarise themselves with the site will encounter any major problems. However, doctors are often busy and impatient, and may not devote the effort to become familiar with the site, and are unlikely to spend much time reading documentation. Users who have initial problems are unlikely to return. So the site should be as easy as possible for first time users.

In addition, the acceptability and usefulness of the site for doctors may depend on minor issues that can only be identified when doctors try using the site, so Tenuta strongly recommended initial in depth pilot testing with a few selected doctors.

The project had also prepared a very complex validation questionnaire. Tenuta recommended that it should be limited to about 10-15 simple questions, as additional questions or a complex questionnaire would significantly reduce the response rate.

Following the visit, Tenuta contacted a doctor by telephone, explained what the site was for, and asked them how they might use it. The doctor was invited to try carrying out their own tasks, and to explain their experiences over the phone. After only a few minutes the doctor commented: "It is so long winded that I would never in a million years use it". There were problems with the complexity of the interface, interpreting the results, and incomplete information in the underlying database. If these findings were replicated with other users, it would confirm the danger that 
validation trials using the existing version of the web site could be a complete disaster!

This highlights the risk of just relying on demonstrating a solution. The way the web site works may make sense to the audience at the time, but nevertheless leave them mystified if they sit down and try to use it unaided.

\section{Conclusions}

Although we have been very successful in raising awareness in the organizations we have worked with, it is difficult to know to what extent our original objective of transferring skills to the projects has been successful. The challenge for future support activities is to find ways to help projects build usability and accessibility into their development processes at an earlier stage. This mirrors a similar challenge to consultants working in industry.

In almost every case we have found that projects have very little awareness of the importance of issues associated with usability and accessibility. In future, we expect the EU to be more demanding when vetting proposals for funding, particularly for near-market projects such as those in the eTEN programme. For example:

- Requirements for usability need to be derived from an in-depth understanding of user needs. As there may be a high risk of product failure in the absence of these requirements, more emphasis should be given to project competence in defining usability requirements, and designing systems that meet these requirements.

- We encountered the common misconception that the best way to get feedback on a service is to demonstrate it to potential users. Projects working with potential users should be required to include one to one usability testing as part of the validation activities.

- There is increasing emphasis within the EU on producing products and services that are accessible to users with disabilities and older users. Legislation already exists in some member states, and a common EU position is likely in the future. In these circumstances one would expect projects to be required to take reasonable steps to develop systems that are accessible.

We did not expect that it would be easy to provide effective support to EU projects, but even we were surprised how difficult it was to gain the initial interest of the projects. Ironically, after we had been invited to provide some support, several projects suggested that Tenuta should be more involved in the projects from their beginning, giving continuous support throughout the lifecycle of the project.

What we discovered working with projects was rather what we expected: very little consideration had been given to how to make project e-services usable and accessible.

But we have had consistently positive feedback on the benefits provided by our support:

- "A new insight into the user experience has been provided."

- "Enabled us to revise the portal with new layout and improved interaction mechanism." 
- "The usability evaluation has been very helpful in discovering new functionalities that should be supported by the software products."

- "Focused the work on the most critical aspects, in order to reach the project objectives."

As this could have a major impact on the eventual commercial success of the planned services, it is important for the EU to have more stringent criteria for competency in this area as part of the conditions of their funding.

\section{References}

1. Bevan, N., Claridge, N., Petrie, H.: Tenuta: Simplified Guidance for Usability and Accessibility. Human Computer International 2005. In: Proceedings HCI International 2005, Lawrence Erlbaum, Mahwah, NJ (2005)

2. Disability Rights Commission: The Web: Access and inclusion for disabled people. London: The Stationery Office (2004) Available at: http://www.drc-gb.org/library/formal_ investigation_report_w.aspx

3. eTEN programme: http://ec.europa.eu/information_society/activities/eten

4. Petrie, H., King, N., Hamilton, F.: Accessibility of museum, library and archive websites: the MLA audit. London: Museums, Libraries and Archives Council (2005) Available at: http://www.mla.gov.uk/resources/assets//M/mla_web_accessibility_pdf_6541.pdf

5. Tenuta support action. Available at: http://www.etenuta.org

6. U.S Department of Health and Human Sciences: Research-Based Web Design \& Usability Guidelines (2006) Available at: http://www.usability.gov/guidelines/

7. Vision Australia: Web Accessibility Toolbar. Available at: http://www.visionaustralia.org.au/ais/ toolbar/

8. W3C, Web Content Accessibility Guidelines 1.0 (1999) Available at: http://www.w3.org/TR/WCAG10/ 